\title{
Empirical Likelihood Inference for First-Order Random Coefficient Integer-Valued Autoregressive Processes
}

\author{
Zhiwen Zhao and Wei Yu \\ College of Mathematics, Jilin Normal University, Siping 136000, China \\ Correspondence should be addressed to Zhiwen Zhao; zhaozhiwenjilin@126.com
}

Received 21 June 2015; Accepted 4 November 2015

Academic Editor: Mustafa Tutar

Copyright (c) 2016 Z. Zhao and W. Yu. This is an open access article distributed under the Creative Commons Attribution License, which permits unrestricted use, distribution, and reproduction in any medium, provided the original work is properly cited.

We apply the empirical likelihood method to estimate the variance of random coefficient in the first-order random coefficient integer-valued autoregressive (RCINAR(1)) processes. The empirical likelihood ratio statistic is derived and some asymptotic theory for it is presented. Furthermore, a simulation study is presented to demonstrate the performance of the proposed method.

\section{Introduction}

Integer-valued time series data are fairly common in practice. Especially in economics and medicine, many interesting variables are integer-valued. In the last three decades, integervalued time series have received increasing attention because of their wide applicability in many different areas, and there were many developments in the literature on it. See, for instance, Davis et al. [1] and MacDonald and Zucchini [2]. For count data, so far there are two main classes of time series models that have been developed in recent years: state-space models and thinning models. For state-space models, we refer to Fukasawa and Basawa [3]. Integer-valued autoregressive (INAR(1)) model was first defined by Steutel and Harn [4] through the "thinning" operator $\circ$. Recall the definition of a "thinning" operator $\circ$ :

$$
\phi \circ X=\sum_{i=1}^{X} B_{i},
$$

where $X$ is an integer-valued random variable and $\phi \in[0,1]$ and $B_{i}$ is an i.i.d. Bernoulli random sequence with $P\left(B_{i}=1\right)=$ $\phi$ that is independent of $X$. Based on the "thinning" operator $\circ$, the INAR(1) model is defined as

$$
X_{t}=\phi \circ X_{t-1}+Z_{t}, \quad t \geq 1,
$$

where $\left\{Z_{t}\right\}$ is a sequence of i.i.d. nonnegative integer-valued random variables.
Note that the parameter $\phi$ may be random and it may vary with time; Zheng et al. [5] introduced the following first-order random coefficient integer-valued autoregressive (RCINAR(1)) model:

$$
X_{t}=\phi_{t} \circ X_{t-1}+Z_{t}, \quad t \geq 1,
$$

where $\left\{\phi_{t}\right\}$ is an independent identically distributed sequence with cumulative distribution function $p_{\phi}$ on $[0,1)$ with $E\left(\phi_{t}\right)=\phi$ and $\operatorname{Var}\left(\phi_{t}\right)=\sigma_{\phi}^{2} ;\left\{Z_{t}\right\}$ is a sequence of i.i.d. nonnegative integer-valued random variables with $E\left(Z_{t}\right)=\lambda$ and $\operatorname{Var}\left(Z_{t}\right)=\sigma_{Z}^{2}$. Moreover, $\left\{\phi_{t}\right\}$ and $\left\{Z_{t}\right\}$ are independent.

Zheng et al. [6] further generalized the above model to the $p$-order cases. In recent several years, RCINAR model has been studied by many authors (see references in [7-10]). In this paper, we are concerned with estimating the variance $\sigma_{\phi}^{2}$ of random coefficient in model (3). We propose an empirical log-likelihood ratio statistics for $\sigma_{\phi}^{2}$ and derive its asymptotic distribution which is standard $\chi^{2}$.

As a nonparametric statistical method, the empirical likelihood method was introduced by Owen [11-13]. The advantages of the empirical likelihood are now widely recognized. It has sampling properties similar to the bootstrap. Many advantages of the empirical likelihood over the normal approximation-based method have also been shown in the literature. These attractive properties have motivated various authors to extend empirical likelihood methodology to other 
situations. Now, the empirical likelihood methods have been widely applied to the statistical inference of the time series models (see [14-21]).

The remainder of this paper is organized as follows: In Section 2, we introduce the methodology and the main results. Simulation results are given in Section 3. Section 4 provides the proofs of the main results.

Throughout the paper, we use the notations “ $\stackrel{d}{\rightarrow}$ " and “ $\rightarrow$ " to denote convergence in distribution and convergence in probability, respectively. Convergence "almost surely" is written as "a.s." Furthermore, $A_{k \times p}^{\tau}$ denotes the transpose matrix of the $k \times p$ matrix $B_{k \times p}$, and $\|\cdot\|$ denotes Euclidean norm of the matrix or vector.

\section{Methodology and Main Results}

In this section, we will first discuss how to apply the empirical likelihood method to estimate the unknown parameter $\sigma_{\phi}^{2}$.

Let $\theta=\left(\phi(1-\phi)-\sigma_{\phi}^{2}, \sigma_{Z}^{2}\right)^{\tau}, \beta=\left(\sigma_{\phi}^{2}, \theta^{\tau}\right)^{\tau}$ and $R_{t}(\phi, \lambda)=$ $X_{t}-E\left(X_{t} \mid X_{t-1}\right)$. For simplicity of notation, we write $R_{t}(\phi, \lambda)$ as $R_{t}$; parameters $\phi$ and $\lambda$ will be omitted. Then, after simple algebra, we get $E\left(X_{t} \mid X_{t-1}\right)=\phi X_{t-1}+\lambda$ and $E\left(R_{t}^{2} \mid X_{t-1}\right)=$ $Z_{t}^{\tau} \beta$, where $Z_{t}=\left(X_{t-1}^{2}, X_{t-1}, 1\right)^{\tau}$.

First we consider estimating $\beta$ by using the conditional least-squares method. Based on the sample $X_{0}, X_{1}, \ldots, X_{n}$, the least-squares estimator $\widehat{\beta}$ of $\beta$ can be obtained by minimizing

$$
Q=\sum_{t=1}^{n}\left(R_{t}^{2}-E\left(R_{t}^{2} \mid X_{t-1}\right)\right)^{2}
$$

with $\beta$. Solving the equation

$$
\frac{\partial Q}{\partial \beta}=\sum_{t=1}^{n}\left(R_{t}^{2}-E\left(R_{t}^{2} \mid X_{t-1}\right)\right) Z_{t}
$$

for $\beta$, we have

$$
\widehat{\beta}=\left(\sum_{t=1}^{n} Z_{t} Z_{t}^{\tau}\right)^{-1} \sum_{t=1}^{n} R_{t}^{2} Z_{t}
$$

Let $\widetilde{\beta}=\widehat{\beta}(\widehat{\phi}, \widehat{\lambda})$, where $\widehat{\phi}$ and $\hat{\lambda}$ are given by Zheng et al. [5]. Further let $\widetilde{\theta}=(0,1,1) \tilde{\beta}$ and $\beta^{*}=\left(\sigma_{\phi}^{2}, \tilde{\theta}^{\tau}\right)^{\tau}$. Then, the estimating equation of $\theta$ can be written as

$$
\sum_{t=1}^{n} T^{\tau}\left(\frac{1}{n} \sum_{t=1}^{n} Z_{t} Z_{t}^{\tau}\right)^{-1} Z_{t}\left(R_{t}^{2}(\widehat{\phi}, \widehat{\lambda})-Z_{t}^{\tau} \beta^{*}\right)=0,
$$

where $T=(1,0,0)^{\tau}$.

In what follows, we apply Owen's empirical likelihood method to make inference about $\sigma_{\phi}^{2}$. For convenience of writing, let

$$
H_{t}\left(\sigma_{\phi}^{2}\right)=T^{\tau}\left(\frac{1}{n} \sum_{t=1}^{n} Z_{t} Z_{t}^{\tau}\right)^{-1} Z_{t}\left(R_{t}^{2}(\widehat{\phi}, \widehat{\lambda})-Z_{t}^{\tau} \beta^{*}\right) ;
$$

$p=\left(p_{1}, \ldots, p_{n}\right)^{\tau}$ be a probability vector with $\sum_{t=1}^{n} p_{t}=1$ and $p_{t} \geq 0$; also, let $\sigma_{\phi 0}^{2}$ denote the true parameter value for $\sigma_{\phi}^{2}$. The log empirical likelihood ratio evaluated at $\sigma_{\phi}^{2}$, a candidate value of $\sigma_{\phi 0}^{2}$, is

$$
l\left(\sigma_{\phi}^{2}\right)=-2 \max _{\sum_{t=1}^{n} p_{t} H_{t}\left(\sigma_{\phi}^{2}\right)=0} \sum_{t=1}^{n} \log \left(n p_{t}\right) .
$$

By using the Lagrange multiplier method, introducing a Lagrange multiplier $\lambda \in R$, we have

$$
l\left(\sigma_{\phi}^{2}\right)=2 \sum_{t=1}^{n} \log \left(1+\lambda^{\tau} H_{t}\left(\sigma_{\phi}^{2}\right)\right)
$$

where $\lambda$ satisfies

$$
\frac{1}{n} \sum_{t=1}^{n} \frac{H_{t}\left(\sigma_{\phi}^{2}\right)}{1+\lambda^{\tau} H_{t}\left(\sigma_{\phi}^{2}\right)}=0 .
$$

Owen's empirical log-likelihood ratio statistic has a chisquared limiting distribution. Similarly, we can prove that $l\left(\sigma_{\phi}^{2}\right)$ will also be asymptotically chi-squared distributed. In order to establish a theory for $l\left(\sigma_{\phi}^{2}\right)$, we assume that the following assumptions hold:

$\left(\mathbf{A}_{1}\right)\left\{X_{t}\right\}$ is a strictly stationary and ergodic process.

$\left(\mathbf{A}_{2}\right) E\left|X_{t}\right|^{8}<\infty$.

Remark 1. Similar conditions can be found in [8].

Now we can give the limiting properties of $l\left(\sigma_{\phi}^{2}\right)$.

Theorem 2. Assume that $\left(\mathbf{A}_{1}\right)$ and $\left(\mathbf{A}_{2}\right)$ hold. If $\sigma_{\phi 0}^{2}$ is the true value of $\sigma_{\phi}^{2}$, then

$$
l\left(\sigma_{\phi 0}^{2}\right) \stackrel{d}{\rightarrow} \chi_{1}^{2} \quad \text { as } n \longrightarrow \infty
$$

where $\chi_{1}^{2}$ is a chi-squared distribution with 1 degree of freedom.

As a consequence of the theorem, confidence regions for the parameter $\sigma_{\phi}^{2}$ can be constructed by (12). For $0<\delta<1$, an asymptotic $100(1-\delta) \%$ confidence region for $\sigma_{\phi}^{2}$ is given by

$$
\mathscr{C}_{\sigma_{\phi}^{2}}\{\delta\}=\left\{\sigma_{\phi}^{2} \in R: l\left(\sigma_{\phi}^{2}\right) \leq \chi_{1}^{2}(\delta)\right\},
$$

where $\chi_{1}^{2}(\delta)$ is the upper $\delta$-quantile of the chi-squared distribution with degrees of freedom equal to 1 .

\section{Simulation Study}

In this section, we conduct some simulation studies which show that our proposed methods perform very well.

In the first simulation study, we consider the RCINAR(1) process:

$$
X_{t}=\phi_{t} \circ X_{t-1}+Z_{t}, \quad t \geq 1,
$$


TABLE 1: Coverage probabilities of the confidence intervals on $\sigma_{\phi}^{2}$.

\begin{tabular}{ccccc}
\hline & $\phi$ & $n=50$ & $n=100$ & $n=300$ \\
\hline \multirow{4}{*}{$\lambda=1$} & 0.1 & $0.980(0.960)$ & $0.976(0.949)$ & $0.979(0.956)$ \\
& 0.2 & $0.972(0.946)$ & $0.984(0.971)$ & $0.984(0.966)$ \\
& 0.3 & $0.977(0.958)$ & $0.986(0.959)$ & $0.990(0.970)$ \\
& 0.4 & $0.983(0.943)$ & $0.984(0.958)$ & $0.984(0.968)$ \\
& 0.5 & $0.978(0.963)$ & $0.986(0.966)$ & $0.990(0.977)$ \\
\hline \multirow{4}{*}{$\lambda=2$} & 0.1 & $0.980(0.952)$ & $0.972(0.943)$ & $0.970(0.975)$ \\
& 0.2 & $0.977(0.956)$ & $0.980(0.980)$ & $0.973(0.979)$ \\
& 0.3 & $0.989(0.961)$ & $0.983(0.967)$ & $0.969(0.978)$ \\
& 0.4 & $0.983(0.969)$ & $0.983(0.966)$ & $0.974(0.981)$ \\
& 0.5 & $0.973(0.968)$ & $0.982(0.965)$ & $0.970(0.971)$ \\
\hline
\end{tabular}

where $\left\{\phi_{t}\right\}$ is a sequence of i.i.d. sequence with $E\left(\phi_{t}\right)=\phi$ and $\operatorname{Var}\left(\phi_{t}\right)=\sigma_{\phi}^{2} ; Z_{t} \sim \operatorname{Poisson}(\lambda)$. We take $\phi=0.1,0.2,0.3,0.4$ and 0.5 and take $\lambda=1$ and 2. Samples of size $n=50,100$ and 300 . All simulation studies are based on 1000 repetitions. The results of the simulations are presented in Table 1. The nominal confidence level is chosen to be 0.90 and 0.95 , and the figures in parentheses are the simulation results at the nominal level of 0.90 .

From Table 1, we find that the confidence region obtained by using the empirical likelihood method has high coverage levels for different $\sigma_{\phi}^{2}$. The coverage probability has no obvious change for different $\phi$ and $\lambda$. That means that the empirical likelihood method is also robust.

In the second simulation study, we illustrate how our method can be applied to fit a set of data through a practical example. We apply model (3) to fit the number of large- and medium-sized civil Boeing 767 cargo planes over the period 1985-2013 in China. The data in Table 2 are provided by the National Bureau of Statistics of China (http://data.stats.gov $. \mathrm{cn} /$ easyquery.htm? $\mathrm{cn}=\mathrm{C} 01)$. The fitting procedure is as follows: Firstly, by using the data over the period 1985-2003, we obtain the estimator of the model parameter. Then, by using this model, we can obtain a fitting sequence over the period 2004-2013. Furthermore, in order to compare with the ordinary autoregressive $(\mathrm{AR}(1))$ model, we also give the fitting results of the $\mathrm{AR}(1)$ model. Table 3 reports the fitting results. In Table 3, Number is the true value and RCINAR(1) and $\operatorname{AR}(1)$ are the fitting results obtained by the RCINAR(1) model and AR(1) model, respectively. For the simulation results of $\mathrm{AR}(1)$ model, we take the rounded integer values of the simulation results. From the simulation results, we can find that the RCINAR(1) model has more plausible fitting results than the $\mathrm{AR}(1)$ model.

\section{Proofs of the Main Results}

Lemma 3. Assume that $\left(\mathbf{A}_{1}\right)$ and $\left(\mathbf{A}_{2}\right)$ hold. Then

$$
\frac{1}{\sqrt{n}} \sum_{t=1}^{n} H_{t}\left(\sigma_{\phi}^{2}\right) \stackrel{d}{\rightarrow} N\left(0, T^{\tau} \Gamma^{-1} W \Gamma^{-1} T\right),
$$

where $W=E\left(Z_{t} Z_{t}^{\tau}\left(R_{t}^{2}-Z_{t}^{\tau} \beta\right)^{2}\right)$ and $\Gamma=E\left(Z_{t} Z_{t}^{\tau}\right)$.
TABLE 2: The number of Boeing 767 cargo planes.

\begin{tabular}{lcccccc}
\hline Year & 1985 & 1986 & 1987 & 1988 & 1989 & 1990 \\
Number & 2 & 2 & 4 & 5 & 6 & 6 \\
\hline Year & 1991 & 1992 & 1993 & 1994 & 1995 & 1996 \\
Number & 6 & 10 & 12 & 16 & 17 & 17 \\
\hline Year & 1997 & 1998 & 1999 & 2000 & 2001 & 2002 \\
Number & 17 & 15 & 16 & 16 & 17 & 18 \\
\hline Year & 2003 & 2004 & 2005 & 2006 & 2007 & 2008 \\
Number & 22 & 27 & 27 & 29 & 22 & 22 \\
\hline Year & 2009 & 2010 & 2011 & 2012 & 2013 & \\
Number & 19 & 18 & 15 & 13 & 11 & \\
\hline
\end{tabular}

TABLE 3: The fitting results.

\begin{tabular}{lcccccc}
\hline Year & 2004 & 2005 & 2006 & 2007 & 2008 & 2009 \\
Number & 27 & 27 & 29 & 22 & 22 & 19 \\
RCINAR(1) & 23 & 22 & 22 & 21 & 19 & 18 \\
AR(1) & 22 & 22 & 23 & 23 & 23 & 25 \\
\hline Year & 2010 & 2011 & 2012 & 2013 & & \\
Number & 18 & 15 & 13 & 11 & & \\
RCINAR(1) & 18 & 18 & 16 & 16 & & \\
AR(1) & 25 & 27 & 27 & 29 & & \\
\hline
\end{tabular}

Proof. Note that

$$
\begin{aligned}
& \frac{1}{\sqrt{n}} \sum_{t=1}^{n} H_{t}\left(\sigma_{\phi}^{2}\right)=T^{\tau}\left(\frac{1}{n} \sum_{t=1}^{n} Z_{t} Z_{t}^{\tau}\right)^{-1} \frac{1}{\sqrt{n}} \\
& \quad \cdot \sum_{t=1}^{n} Z_{t}\left(R_{t}^{2}(\widehat{\phi}, \widehat{\lambda})-Z_{t}^{\tau} \beta\right)=T^{\tau}\left(\frac{1}{n} \sum_{t=1}^{n} Z_{t} Z_{t}^{\tau}\right)^{-1} \frac{1}{\sqrt{n}} \\
& \quad \cdot \sum_{t=1}^{n} Z_{t}\left(R_{t}^{2}(\widehat{\phi}, \widehat{\lambda})-R_{t}^{2}(\phi, \lambda)\right) \\
& \quad+T^{\tau}\left(\frac{1}{n} \sum_{t=1}^{n} Z_{t} Z_{t}^{\tau}\right)^{-1} \frac{1}{\sqrt{n}} \sum_{t=1}^{n} Z_{t}\left(R_{t}^{2}(\phi, \lambda)-Z_{t}^{\tau} \beta\right) \\
& \quad+T^{\tau}\left(\frac{1}{n} \sum_{t=1}^{n} Z_{t} Z_{t}^{\tau}\right)^{-1} \frac{1}{\sqrt{n}} \sum_{t=1}^{n} Z_{t} Z_{t}^{\tau}\left(\beta-\beta^{*}\right) \triangleq A_{n 1} \\
& \quad+A_{n 2}+A_{n 3} .
\end{aligned}
$$

First, we consider $A_{n 3}$. After simple algebra calculation, we have

$$
\begin{aligned}
A_{n 3} & =T^{\tau}\left(\frac{1}{n} \sum_{t=1}^{n} Z_{t} Z_{t}^{\tau}\right)^{-1} \frac{1}{\sqrt{n}} \sum_{t=1}^{n} Z_{t} Z_{t}^{\tau}\left(\beta-\beta^{*}\right) \\
& =T^{\tau}\left(\frac{1}{n} \sum_{t=1}^{n} Z_{t} Z_{t}^{\tau}\right)^{-1}\left(\frac{1}{n} \sum_{t=1}^{n} Z_{t} Z_{t}^{\tau}\right) \sqrt{n}\left(\beta-\beta^{*}\right) \\
& =\sqrt{n} T^{\tau}\left(\beta-\beta^{*}\right)=0 .
\end{aligned}
$$



have

Next, we consider $A_{n 1}$. By the mean value theorem, we

$$
\begin{aligned}
& R_{t}^{2}(\widehat{\phi}, \widehat{\lambda})-R_{t}^{2}(\phi, \lambda) \\
& \quad=-2 R_{t}\left(\phi^{*}, \lambda^{*}\right)\left(X_{t-1}(\widehat{\phi}-\phi)+\widehat{\lambda}-\lambda\right),
\end{aligned}
$$

where $\phi^{*}$ lies between $\widehat{\phi}$ and $\phi$ and $\lambda^{*}$ lies between $\hat{\lambda}$ and $\lambda$. Therefore,

$$
\begin{aligned}
& A_{n 1}=T^{\tau}\left(\frac{1}{n} \sum_{t=1}^{n} Z_{t} Z_{t}^{\tau}\right)^{-1} \frac{1}{\sqrt{n}} \\
& \cdot \sum_{t=1}^{n} Z_{t}\left(R_{t}^{2}(\widehat{\phi}, \widehat{\lambda})-R_{t}^{2}(\phi, \lambda)\right) \\
& =T^{\tau}\left(\frac{1}{n} \sum_{t=1}^{n} Z_{t} Z_{t}^{\tau}\right)^{-1} \frac{-2}{\sqrt{n}} \\
& \cdot \sum_{t=1}^{n} Z_{t} R_{t}\left(\phi^{*}, \lambda^{*}\right)\left(X_{t-1}(\widehat{\phi}-\phi)+\widehat{\lambda}-\lambda\right) \\
& =T^{\tau}\left(\frac{1}{n} \sum_{t=1}^{n} Z_{t} Z_{t}^{\tau}\right)^{-1} \frac{-2}{\sqrt{n}} \\
& \cdot \sum_{t=1}^{n} Z_{t}\left(R_{t}(\phi, \lambda)+R_{t}\left(\phi^{*}, \lambda^{*}\right)-R_{t}(\phi, \lambda)\right) \\
& \cdot\left(X_{t-1}(\widehat{\phi}-\phi)+\widehat{\lambda}-\lambda\right)=T^{\tau}\left(\frac{1}{n} \sum_{t=1}^{n} Z_{t} Z_{t}^{\tau}\right)^{-1} \frac{-2}{\sqrt{n}} \\
& \cdot \sum_{t=1}^{n} Z_{t}\left(\left(\phi-\phi^{*}\right) X_{t-1}+\lambda-\lambda^{*}+R_{t}(\phi, \lambda)\right) \\
& \cdot\left(X_{t-1}(\widehat{\phi}-\phi)+\widehat{\lambda}-\lambda\right)=T^{\tau}\left(\frac{1}{n} \sum_{t=1}^{n} Z_{t} Z_{t}^{\tau}\right)^{-1} \frac{-2}{\sqrt{n}} \\
& \cdot \sum_{t=1}^{n} Z_{t} R_{t}(\phi, \lambda) X_{t-1}(\widehat{\phi}-\phi)-T^{\tau}\left(\frac{1}{n} \sum_{t=1}^{n} Z_{t} Z_{t}^{\tau}\right)^{-1} \\
& \frac{2}{\sqrt{n}} \sum_{t=1}^{n} Z_{t} R_{t}(\phi, \lambda)(\widehat{\lambda}-\lambda)-T^{\tau}\left(\frac{1}{n} \sum_{t=1}^{n} Z_{t} Z_{t}^{\tau}\right)^{-1} \\
& \cdot \frac{2}{\sqrt{n}} \sum_{t=1}^{n} Z_{t}\left(\phi-\phi^{*}\right) X_{t-1}^{2}(\widehat{\phi}-\phi) \\
& -T^{\tau}\left(\frac{1}{n} \sum_{t=1}^{n} Z_{t} Z_{t}^{\tau}\right)^{-1} \frac{2}{\sqrt{n}} \\
& \cdot \sum_{t=1}^{n} Z_{t}\left(\lambda-\lambda^{*}\right) X_{t-1}(\widehat{\phi}-\phi)-T^{\tau}\left(\frac{1}{n} \sum_{t=1}^{n} Z_{t} Z_{t}^{\tau}\right)^{-1} \\
& \frac{2}{\sqrt{n}} \sum_{t=1}^{n} Z_{t}\left(\phi-\phi^{*}\right) X_{t-1}(\hat{\lambda}-\lambda)
\end{aligned}
$$

Below, we prove that $B_{n i}=o_{p}(1), i=1,2,3,4,5,6$. For $B_{n 1}$, note that

$$
\begin{aligned}
B_{n 1}= & T^{\tau}\left(\frac{1}{n} \sum_{t=1}^{n} Z_{t} Z_{t}^{\tau}\right)^{-1} \frac{-2}{n} \\
& \cdot \sum_{t=1}^{n} Z_{t} R_{t}(\phi, \lambda) X_{t-1}(\sqrt{n}(\widehat{\phi}-\phi)) .
\end{aligned}
$$

By Theorem 3.1 in Zheng et al. [5], we know that

$$
\sqrt{n}(\widehat{\phi}-\phi)=O_{p}(1) \text {. }
$$

Moreover, by the ergodic theorem, we have

$$
\begin{gathered}
\frac{-2}{n} \sum_{t=1}^{n} Z_{t} R_{t}(\phi, \lambda) X_{t-1} \stackrel{p}{\rightarrow}-2 E\left(Z_{t} R_{t}(\phi, \lambda) X_{t-1}\right), \\
\frac{1}{n} \sum_{t=1}^{n} Z_{t} Z_{t}^{\tau} \stackrel{p}{\rightarrow} \Gamma .
\end{gathered}
$$

Further note that

$$
\begin{aligned}
E & \left(Z_{t} R_{t}(\phi, \lambda) X_{t-1}\right) \\
& =E\left(E\left(\left(R_{t}(\phi, \lambda) Z_{t} X_{t-1}\right) \mid \mathscr{F}_{t-1}\right)\right) \\
& =E\left(Z_{t} X_{t-1} E\left(R_{t}(\phi, \lambda) \mid \mathscr{F}_{t-1}\right)\right)=0,
\end{aligned}
$$

which, combined with (22) and (23), implies that

$$
B_{n 1}=O_{p}(1) o_{p}(1) O_{p}(1)=o_{p}(1) .
$$

Similarly, we can prove that

$$
B_{n 2}=o_{p}(1)
$$

Next, we prove that

$$
B_{n 3}=o_{p}(1)
$$

Note that

$$
\begin{aligned}
& \left\|B_{n 3}\right\| \\
& \leq\left\|\frac{1}{n} \sum_{t=1}^{n} Z_{t} Z_{t}^{\tau}\right\| \frac{2}{\sqrt{n}} \sum_{t=1}^{n}\left\|Z_{t} X_{t-1}^{2}\right\|\left\|\phi-\phi^{*}\right\|\|\hat{\phi}-\phi\| \\
& \leq\left\|\frac{1}{n} \sum_{t=1}^{n} Z_{t} Z_{t}^{\tau}\right\| \frac{2}{\sqrt{n}} \sum_{t=1}^{n}\left|Z_{t} X_{t-1}^{2}\right|\left\|\phi-\phi^{*}\right\|^{2} \\
& \leq\left\|\frac{1}{n} \sum_{t=1}^{n} Z_{t} Z_{t}^{\tau}\right\| \frac{1}{\sqrt{n}} \frac{2}{n} \sum_{t=1}^{n}\left|Z_{t} X_{t-1}^{2}\right|(\|\sqrt{n}(\hat{\phi}-\phi)\|)^{2} .
\end{aligned}
$$


By the ergodic theorem, we have

$$
\frac{2}{n} \sum_{t=1}^{n}\left|Z_{t} X_{t-1}^{2}\right|=O_{p}(1)
$$

By (21), we have

$$
\|\sqrt{n}(\widehat{\phi}-\phi)\|^{2}=O_{p}(1)
$$

Therefore, by (21), we have

$$
B_{n 3}=O_{p}(1) o_{p}(1) O_{p}(1) O_{p}(1)=o_{p}(1) .
$$

Similarly, we can prove that

$$
B_{n i}=o_{p}(1), \quad i=4,5,6 .
$$

Using this, together with (25), (26), and (27), we can prove

$$
A_{n 1}=o_{p}(1) \text {. }
$$

Finally, we prove that

$$
A_{n 2} \stackrel{d}{\rightarrow} N\left(0, T^{\tau} \Gamma^{-1} W \Gamma^{-1} T\right)
$$

For this, we first prove that

$$
\frac{1}{\sqrt{n}} \sum_{t=1}^{n} Z_{t}\left(R_{t}^{2}-Z_{t}^{\tau} \beta\right) \stackrel{d}{\rightarrow} N(0, W) .
$$

By the Cramer-Wold device, it suffices to show that, for all $c \in R^{3} \backslash(0,0,0)$,

$$
\frac{1}{\sqrt{n}} \sum_{t=1}^{n} c^{\tau} Z_{t}\left(R_{t}^{2}-Z_{t}^{\tau} \beta\right) \stackrel{d}{\rightarrow} N\left(0, c^{\tau} W c\right) .
$$

Let $\xi_{n t}=(1 / \sqrt{n}) c^{\tau} Z_{t}\left(R_{t}^{2}-Z_{t}^{\tau} \beta\right)$ and $\mathscr{F}_{n t}=\sigma\left(\xi_{n r}, 1 \leq r \leq t\right)$. Then $\left\{\sum_{t=1}^{n} \xi_{n t}, \mathscr{F}_{n t}, 1 \leq t \leq n, n \geq 1\right\}$ is a zero-mean, square integrable martingale array. By making use of a martingale central limit theorem [22], we can prove (36). Further, by (23), we know that (34) holds. Therefore, by (17), (33), and (34), we can prove Lemma 3.

Lemma 4. Assume that $\left(\mathbf{A}_{1}\right)$ and $\left(\mathbf{A}_{2}\right)$ hold. Then

$$
\frac{1}{n} \sum_{t=1}^{n} H_{t}^{2}\left(\sigma_{\phi}^{2}\right) \stackrel{p}{\rightarrow} T^{\tau} \Gamma^{-1} W \Gamma^{-1} T .
$$

Proof. Note that

$$
\begin{aligned}
\frac{1}{n} \sum_{t=1}^{n} H_{t}^{2}\left(\sigma_{\phi}^{2}\right)= & T^{\tau}\left(\frac{1}{n} \sum_{t=1}^{n} Z_{t} Z_{t}^{\tau}\right)^{-1} \frac{1}{n} \\
& \cdot \sum_{t=1}^{n} Z_{t} Z_{t}^{\tau}\left(R_{t}^{2}(\widehat{\phi}, \widehat{\lambda})-Z_{t}^{\tau} \beta^{*}\right)^{2} \\
& \cdot\left(\frac{1}{n} \sum_{t=1}^{n} Z_{t} Z_{t}^{\tau}\right)^{-1} T .
\end{aligned}
$$

By (23), in order to prove Lemma 4, we have only to show that

$$
\frac{1}{n} \sum_{t=1}^{n} Z_{t} Z_{t}^{\tau}\left(R_{t}^{2}(\widehat{\phi}, \widehat{\lambda})-Z_{t}^{\tau} \beta^{*}\right)^{2} \stackrel{p}{\rightarrow} W .
$$

Note that

$$
\begin{aligned}
& \frac{1}{n} \sum_{t=1}^{n} Z_{t} Z_{t}^{\tau}\left(R_{t}^{2}(\widehat{\phi}, \hat{\lambda})-Z_{t}^{\tau} \beta^{*}\right)^{2}=\frac{1}{n} \\
& \quad \cdot \sum_{t=1}^{n} Z_{t} Z_{t}^{\tau}\left(R_{t}^{2}(\widehat{\phi}, \widehat{\lambda})-R_{t}^{2}(\phi, \lambda)+R_{t}^{2}(\phi, \lambda)-Z_{t}^{\tau} \beta\right. \\
& \left.\quad+Z_{t}^{\tau} \beta-Z_{t}^{\tau} \beta^{*}\right)^{2}=\frac{1}{n} \sum_{t=1}^{n} Z_{t} Z_{t}^{\tau}\left(R_{t}^{2}(\phi, \lambda)-Z_{t}^{\tau} \beta\right)^{2} \\
& \quad+\frac{1}{n} \sum_{t=1}^{n} Z_{t} Z_{t}^{\tau}\left(R_{t}^{2}(\widehat{\phi}, \widehat{\lambda})-R_{t}^{2}(\phi, \lambda)\right)^{2}+\frac{1}{n} \\
& \quad \cdot \sum_{t=1}^{n} Z_{t} Z_{t}^{\tau}\left(Z_{t}^{\tau} \beta-Z_{t}^{\tau} \beta^{*}\right)^{2}+\frac{2}{n} \sum_{t=1}^{n} Z_{t} Z_{t}^{\tau}\left(R_{t}^{2}(\widehat{\phi}, \widehat{\lambda})\right. \\
& \left.\quad-R_{t}^{2}(\phi, \lambda)\right)\left(R_{t}^{2}(\phi, \lambda)-Z_{t}^{\tau} \beta\right)+\frac{2}{n} \\
& \quad \cdot \sum_{t=1}^{n} Z_{t} Z_{t}^{\tau}\left(R_{t}^{2}(\widehat{\phi}, \widehat{\lambda})-R_{t}^{2}(\phi, \lambda)\right)\left(Z_{t}^{\tau} \beta-Z_{t}^{\tau} \beta^{*}\right) \\
& \quad+\frac{2}{n} \sum_{t=1}^{n} Z_{t} Z_{t}^{\tau}\left(R_{t}^{2}(\phi, \lambda)-Z_{t}^{\tau} \beta\right)\left(Z_{t}^{\tau} \beta-Z_{t}^{\tau} \beta^{*}\right) \\
& \quad \triangleq C_{n 1}+C_{n 2}+C_{n 3}+C_{n 4}+C_{n 5}+C_{n 6} \cdot
\end{aligned}
$$

By the ergodic theorem, we know that

$$
C_{n 1} \stackrel{p}{\rightarrow} W
$$

Similar to the proof of (33), we can further prove that

$$
C_{n i}=o_{p}(1), \quad i=2,3,4,5,6 .
$$

This, in conjunction with (41), yields (39). So we complete the proof of Lemma 4.

Lemma 5. Assume that $\left(\mathbf{A}_{1}\right)$ and $\left(\mathbf{A}_{2}\right)$ hold. Then

$$
\max _{1 \leq t \leq n}\left|H_{t}\left(\sigma_{\phi}^{2}\right)\right|=o_{p}\left(n^{1 / 2}\right) \text {. }
$$

Proof. To prove (43), we only need to prove that

$$
\frac{1}{n} \max _{1 \leq t \leq n} H_{t}^{2}\left(\sigma_{\phi}^{2}\right) \stackrel{p}{\rightarrow} 0 .
$$

Let $T^{\tau} \Gamma^{-1} W \Gamma^{-1} T=\sigma^{2}$. For $m \in\{1, \ldots, n\}$, define

$$
B_{n, m} \triangleq \bigcap_{j=1}^{n}\left\{\omega:\left|\frac{1}{n} \sum_{t=1}^{[n(j / m)]} H_{t}\left(\sigma_{\phi}^{2}\right)-\frac{j}{m} \sigma^{2}\right| \leq \frac{1}{m}\right\},
$$


where $[n(j / m)]$ denotes the largest integer not greater than $n(j / m)$. For each $m$, (37) implies that $P\left(B_{n, m}\right) \stackrel{P}{\rightarrow} 1$ as $n \rightarrow \infty$. Moreover, note that

$$
\max _{1 \leq t \leq n} \frac{H_{t}\left(\sigma_{\phi}^{2}\right)}{n} \leq \frac{1}{n_{s \in[0,1]}} \sup _{t=[n s]+1}^{[n(s+(1 / m))]} H_{t}\left(\sigma_{\phi}^{2}\right) .
$$

For given $s \in[0,1]$, choose $j \in\{1, \ldots, m\}$ so that $s \in[(j-$ $1) / m, j / m]$. Therefore, for each $s \in[0,1]$, if $\omega \in B_{n, m}$, then we have

$$
\begin{aligned}
\frac{1}{n} \sum_{t=[n s]+1}^{[n(s+(1 / m))]} H_{t}\left(\sigma_{\phi}^{2}\right) \leq \frac{1}{n} \sum_{t=[n(j-1) / m]+1}^{[n(j+1) / m]} H_{t}\left(\sigma_{\phi}^{2}\right) \\
=\left(\frac{1}{n} \sum_{t=1}^{[n(j+1) / m]} H_{t}\left(\sigma_{\phi}^{2}\right)-\frac{j+1}{m} \sigma^{2}\right) \\
\quad-\left(\frac{1}{n} \sum_{t=1}^{[n(j-1) / m]} H_{t}\left(\sigma_{\phi}^{2}\right)-\frac{j-1}{m} \sigma^{2}\right)+\frac{2}{m} \sigma^{2} \\
\leq \frac{2}{m}+\frac{2}{m} \sigma^{2}=\frac{2}{m}\left(1+\sigma^{2}\right) .
\end{aligned}
$$

So, for any $m \geq 1$,

$$
\begin{aligned}
& \lim _{n \rightarrow \infty} P\left\{\max _{1 \leq t \leq n} \frac{H_{t}\left(\sigma_{\phi}^{2}\right)}{n} \leq \frac{2}{m}\left(1+\sigma^{2}\right)\right\} \\
& \geq \lim _{n \rightarrow \infty} P\left(B_{n, m}\right)=1,
\end{aligned}
$$

which implies (44). So we prove (43).

Proof of Theorem 2. First, we prove that

$$
\lambda=O_{p}\left(n^{-1 / 2}\right) .
$$

Write $\lambda=\rho \vartheta$, where $\rho \geq 0$ and $|\vartheta|=1$. Observe that

$$
\begin{aligned}
0 & =\left|\frac{1}{n} \sum_{t=1}^{n} \frac{H_{t}\left(\sigma_{\phi}^{2}\right)}{1+\lambda H_{t}\left(\sigma_{\phi}^{2}\right)}\right|=\left|\vartheta \frac{1}{n} \sum_{t=1}^{n} \frac{H_{t}\left(\sigma_{\phi}^{2}\right)}{1+\lambda H_{t}\left(\sigma_{\phi}^{2}\right)}\right| \\
& =\frac{1}{n}\left|\vartheta\left(\sum_{t=1}^{n} H_{t}\left(\sigma_{\phi}^{2}\right)-\rho \sum_{t=1}^{n} \frac{\vartheta H_{t}^{2}\left(\sigma_{\phi}^{2}\right)}{1+\rho \vartheta H_{t}\left(\sigma_{\phi}^{2}\right)}\right)\right| \\
& \geq \frac{\rho}{n} \vartheta^{2} \sum_{t=1}^{n} \frac{H_{t}^{2}\left(\sigma_{\phi}^{2}\right)}{1+\rho \vartheta H_{t}\left(\sigma_{\phi}^{2}\right)}-\frac{1}{n}\left|\vartheta \sum_{t=1}^{n} H_{t}\left(\sigma_{\phi}^{2}\right)\right| \\
& \geq \frac{\rho}{n} \vartheta^{2} \sum_{t=1}^{n} \frac{H_{t}^{2}\left(\sigma_{\phi}^{2}\right)}{1+\rho \max _{1 \leq t \leq n}\left|H_{t}\left(\sigma_{\phi}^{2}\right)\right|}
\end{aligned}
$$

$$
\begin{aligned}
& -\frac{1}{n}\left|\vartheta \sum_{t=1}^{n} H_{t}\left(\sigma_{\phi}^{2}\right)\right| \\
\geq & \frac{1}{1+\rho \max _{1 \leq t \leq n}\left|H_{t}\left(\sigma_{\phi}^{2}\right)\right|} \rho \vartheta^{2} \frac{1}{n} \sum_{t=1}^{n} H_{t}^{2}\left(\sigma_{\phi}^{2}\right) \\
& -\frac{1}{n}\left|\vartheta \sum_{t=1}^{n} H_{t}\left(\sigma_{\phi}^{2}\right)\right| .
\end{aligned}
$$

This implies that

$$
\begin{aligned}
& \frac{1}{1+\rho \max _{1 \leq t \leq n}\left|H_{t}\left(\sigma_{\phi}^{2}\right)\right|} \rho \vartheta^{2} \frac{1}{n} \sum_{t=1}^{n} H_{t}^{2}\left(\sigma_{\phi}^{2}\right) \\
& \leq \frac{1}{n}\left|\vartheta \sum_{t=1}^{n} H_{t}\left(\sigma_{\phi}^{2}\right)\right| .
\end{aligned}
$$

Further, by Lemma 4, we know that

$$
\vartheta^{2} \frac{1}{n} \sum_{t=1}^{n} H_{t}^{2}\left(\sigma_{\phi}^{2}\right)=O_{p}(1)
$$

By Lemma 3, we have

$$
\frac{1}{n}\left|\vartheta \sum_{t=1}^{n} H_{t}\left(\sigma_{\phi}^{2}\right)\right|=O_{p}\left(n^{-1 / 2}\right) .
$$

Thus by (51) and Lemma 5, we have

$$
\rho=|\lambda|=O_{p}\left(n^{-1 / 2}\right),
$$

which implies (49).

By (49) and Lemma 5, we can prove that

$$
\max _{1 \leq t \leq n}\left|\lambda H_{t}\left(\sigma_{\phi}^{2}\right)\right|=O_{p}\left(n^{-1 / 2}\right) o_{p}\left(n^{1 / 2}\right)=o_{p}(1) .
$$

Expanding (11), we have

$$
\begin{aligned}
0= & \frac{1}{n} \sum_{t=1}^{n} \frac{H_{t}\left(\sigma_{\phi}^{2}\right)}{1+\lambda H_{t}\left(\sigma_{\phi}^{2}\right)} \\
= & \frac{1}{n} \sum_{t=1}^{n} H_{t}\left(\sigma_{\phi}^{2}\right)-\frac{1}{n} \lambda \sum_{t=1}^{n} H_{t}^{2}\left(\sigma_{\phi}^{2}\right) \\
& +\frac{1}{n} \sum_{t=1}^{n} \frac{\lambda^{2} H_{t}^{3}\left(\sigma_{\phi}^{2}\right)}{1+\lambda H_{t}\left(\sigma_{\phi}^{2}\right)} .
\end{aligned}
$$

By (55) and Lemmas 3, 4, and 5, we know that the final term in (56) is bounded by

$$
\begin{aligned}
& \frac{1}{n} \sum_{t=1}^{n}\left|H_{t}\left(\sigma_{\phi}^{2}\right)\right|^{3}|\lambda|^{2}\left|1+\lambda H_{t}\left(\sigma_{\phi}^{2}\right)\right|^{-1} \\
& \quad=o_{p}\left(n^{1 / 2}\right) O_{p}\left(n^{-1}\right) O_{p}(1)=o_{p}\left(n^{-1 / 2}\right) .
\end{aligned}
$$


This, together with (41), yields

$$
\lambda=\left(\frac{1}{n} \sum_{t=1}^{n} H_{t}^{2}\left(\sigma_{\phi}^{2}\right)\right)^{-1} \frac{1}{n} \sum_{t=1}^{n} H_{t}\left(\sigma_{\phi}^{2}\right)+o_{p}\left(n^{-1 / 2}\right) .
$$

By the Taylor expansion, we have

$$
\log \left(1+\lambda H_{t}\left(\sigma_{\phi}^{2}\right)\right)=\lambda H_{t}\left(\sigma_{\phi}^{2}\right)-\frac{\left(\lambda H_{t}\left(\sigma_{\phi}^{2}\right)\right)^{2}}{2}+\varphi_{t}
$$

Below, we prove that there exists a finite number $Q>0$, such that

$$
P\left(\left|\varphi_{t}\right| \leq Q\left|\lambda H_{t}\left(\sigma_{\phi}^{2}\right)\right|^{3}, 1 \leq t \leq n\right) \longrightarrow 1
$$

as $n \longrightarrow \infty$.

The Taylor expansion of $\log (1+x)$ around $x=0$ yields

$$
\log (1+x)=x-\frac{x^{2}}{2}+\frac{x^{3}}{3}+\omega(x)
$$

where, as $x \rightarrow 0, \omega(x) / x^{3} \rightarrow 0$. Thus, there exists $\iota>0$, such that $\left|\omega(x) / x^{3}\right|<1 / 6$ for any $|x|<\iota$. Moreover, by (55), we have

$$
\lim _{n \rightarrow \infty} P\left\{\max _{1 \leq t \leq n}\left|\lambda H_{t}\left(\sigma_{\phi}^{2}\right)\right|^{3}<\iota^{3}\right\}=1
$$

Let $A_{n}=\left\{\omega: \max _{1 \leq t \leq n}\left|\lambda H_{t}\left(\sigma_{\phi}^{2}\right)\right|^{3}<\iota^{3}\right\}$. Note that if $\omega \in A_{n}$, then for $1 \leq t \leq n$,

$$
\begin{aligned}
\frac{\left|\varphi_{t}\right|}{\left|\lambda H_{t}\left(\sigma_{\phi}^{2}\right)\right|^{3}} & =\frac{\left|\left(\lambda H_{t}\left(\sigma_{\phi}^{2}\right)\right)^{3} / 3+\omega\left(\lambda H_{t}\left(\sigma_{\phi}^{2}\right)\right)\right|}{\left|\lambda H_{t}\left(\sigma_{\phi}^{2}\right)\right|^{3}} \\
& \leq \frac{1}{3}+\frac{1}{6}=\frac{1}{2},
\end{aligned}
$$

which implies that

$$
P\left(\left|\varphi_{t}\right| \leq Q\left|\lambda H_{t}\left(\sigma_{\phi}^{2}\right)\right|^{3}, 1 \leq t \leq n\right) \longrightarrow 1
$$

$$
\text { as } n \longrightarrow \infty \text {, }
$$

where $Q=1 / 2$.

Moreover, by (10) and (58), we have

$$
\begin{aligned}
l\left(\sigma_{\phi}^{2}\right)= & \left(\frac{1}{\sqrt{n}} \sum_{t=1}^{n} H_{t}\left(\sigma_{\phi}^{2}\right)\right)\left(\frac{1}{n} \sum_{t=1}^{n} H_{t}^{2}\left(\sigma_{\phi}^{2}\right)\right)^{-1} \\
& \cdot\left(\frac{1}{\sqrt{n}} \sum_{t=1}^{n} H_{t}\left(\sigma_{\phi}^{2}\right)\right)+o_{p}(1) .
\end{aligned}
$$

This, together with Lemmas 3 and 4, implies Theorem 2.

\section{Conflict of Interests}

The authors declare that there is no conflict of interests regarding the publication of this paper.

\section{Acknowledgments}

The authors acknowledge the financial supports by National Natural Science Foundation of China (nos. 11571138, 11271155, 11001105, 11071126, 10926156, and 11071269), Specialized Research Fund for the Doctoral Program of Higher Education (no. 20110061110003), Program for New Century Excellent Talents in University (NCET-08-237), Scientific Research Fund of Jilin University (no. 201100011), and Jilin Province Natural Science Foundation (nos. 20130101066JC, 20130522102JH, and 20101596).

\section{References}

[1] R. A. Davis, T. M. Dunsmuir, and Y. Wang, "Modeling time series of count data," in Asymptotics, Nonparametrics and Time Series, pp. 63-114, Marcel-Dekker, New York, NY, USA, 1999.

[2] I. L. MacDonald and W. Z. Zucchini, Hidden Markov and Other Models for Discrete-Valued Time Series, Chapman \& Hall, London, UK, 1997.

[3] T. Fukasawa and I. V. Basawa, "Estimation for a class of generalized state-space time series models," Statistics \& Probability Letters, vol. 60, no. 4, pp. 459-473, 2002.

[4] F. W. Steutel and K. V. Harn, "Discrete analogues of selfdecomposability and stability," The Annals of Probability, vol. 7, no. 5, pp. 893-899, 1979.

[5] H. Zheng, I. V. Basawa, and S. Datta, "First-order random coefficient integer-valued autoregressive processes," Journal of Statistical Planning and Inference, vol. 137, no. 1, pp. 212-229, 2007.

[6] H. Zheng, I. V. Basawa, and S. Datta, "Inference for pth-order random coefficient integer-valued autoregressive processes," Journal of Time Series Analysis, vol. 27, no. 3, pp. 411-440, 2006.

[7] J. Kang and S. Lee, "Parameter change test for random coefficient integer-valued autoregressive processes with application to polio data analysis," Journal of Time Series Analysis, vol. 30, no. 2, pp. 239-258, 2009.

[8] H. Zhang, D. Wang, and F. Zhu, "The empirical likelihood for first-order random coefficient integer-valued autoregressive processes," Communications in Statistics. Theory and Methods, vol. 40, no. 3, pp. 492-509, 2011.

[9] H. Zhang, D. Wang, and F. Zhu, "Empirical likelihood inference for random coefficient INAR $(p)$ process," Journal of Time Series Analysis, vol. 32, no. 3, pp. 195-203, 2011.

[10] A. Roitershtein and Z. Zhong, "On random coefficient INAR(1) processes," Science China Mathematics, vol. 56, no. 1, pp. 177200, 2013.

[11] A. B. Owen, "Empirical likelihood ratio confidence intervals for a single functional," Biometrika, vol. 75, no. 2, pp. 237-249, 1988.

[12] A. B. Owen, "Empirical likelihood ratio confidence region," The Annals of Statistics, vol. 18, no. 1, pp. 90-120, 1990.

[13] A. B. Owen, "Empirical likelihood for linear models," The Annals of Statistics, vol. 19, no. 4, pp. 1725-1747, 1991.

[14] C.-S. Chuang and N. H. Chan, "Empirical likelihood for autoregressive models, with applications to unstable time series," Statistica Sinica, vol. 12, no. 2, pp. 387-407, 2002.

[15] S. X. Chen, W. Härdle, and M. Li, "An empirical likelihood goodness-of-fit test for time series," Journal of the Royal Statistical Society-Series B: Statistical Methodology, vol. 65, no. 3, pp. 663-678, 2003. 
[16] N. H. Chan and S. Ling, "Empirical likelihood for GARCH models," Econometric Theory, vol. 22, no. 3, pp. 403-428, 2006.

[17] S. X. Chen and J. T. Gao, "An adaptive empirical likelihood test for parametric time series regression models," Journal of Econometrics, vol. 141, no. 2, pp. 950-972, 2007.

[18] P. Guggenberger and R. J. Smith, "Generalized empirical likelihood tests in time series models with potential identification failure," Journal of Econometrics, vol. 142, no. 1, pp. 134-161, 2008.

[19] J. Li, W. Liang, S. He, and X. Wu, "Empirical likelihood for the smoothed LAD estimator in infinite variance autoregressive models," Statistics \& Probability Letters, vol. 80, no. 17-18, pp. 1420-1430, 2010.

[20] Z.-W. Zhao and D.-H. Wang, "Empirical likelihood for an autoregressive model with explanatory variables," Communications in Statistics. Theory and Methods, vol. 40, no. 3, pp. 559570, 2011.

[21] Z.-W. Zhao, D.-H. Wang, and C.-X. Peng, "Coefficient constancy test in generalized random coefficient autoregressive model," Applied Mathematics and Computation, vol. 219, no. 20, pp. 10283-10292, 2013.

[22] P. Hall and C. C. Heyde, Martingale Limit Theory and Its Application, Academic Press, New York, NY, USA, 1980. 


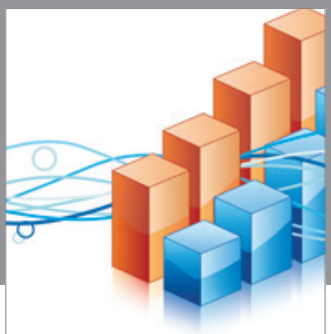

Advances in

Operations Research

vatem alat4

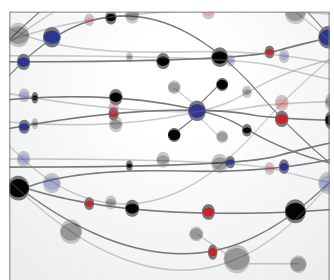

\section{The Scientific} World Journal
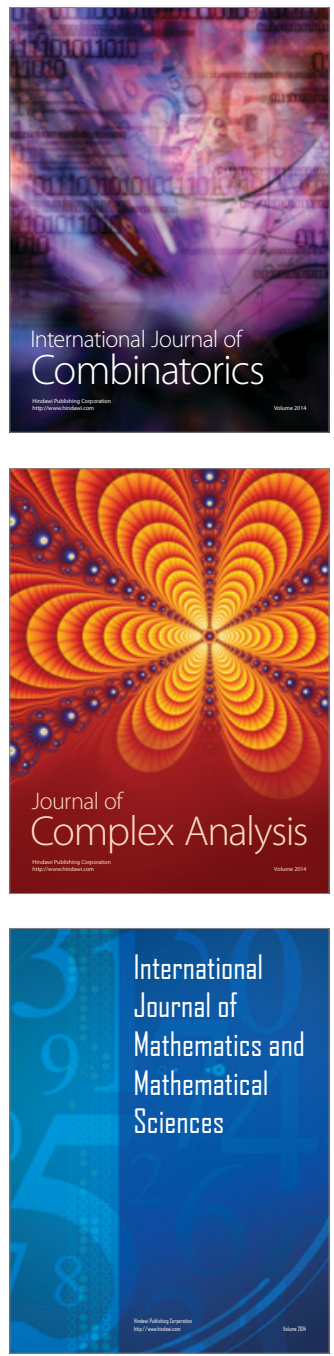
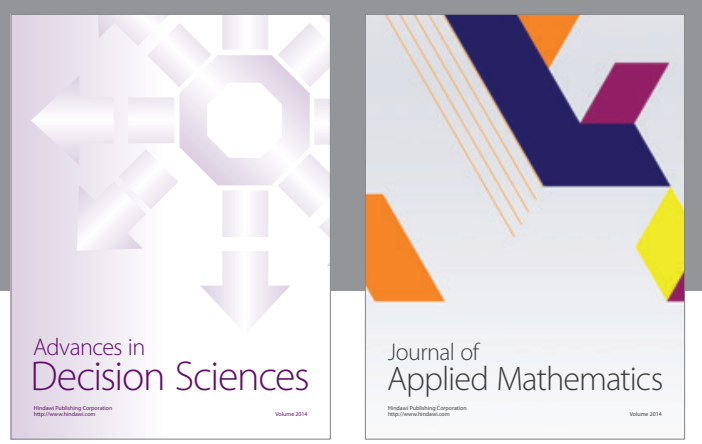

Algebra

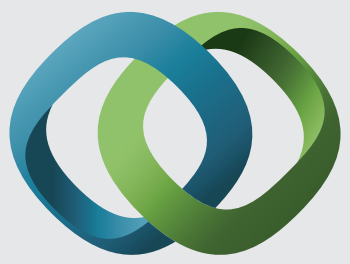

\section{Hindawi}

Submit your manuscripts at

http://www.hindawi.com
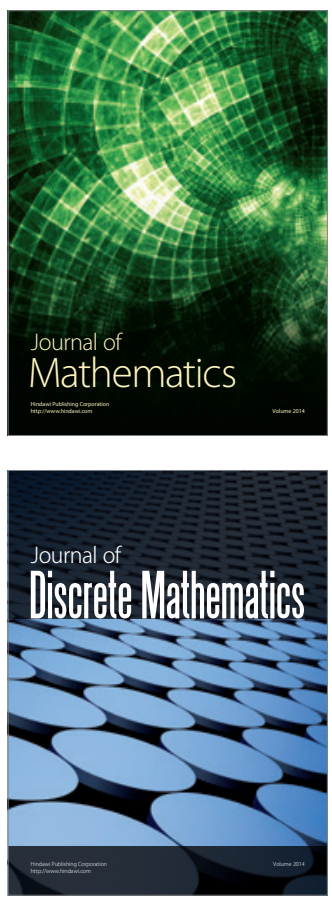

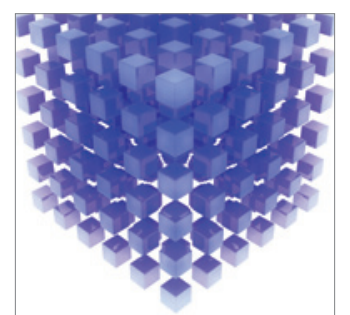

Mathematical Problems in Engineering
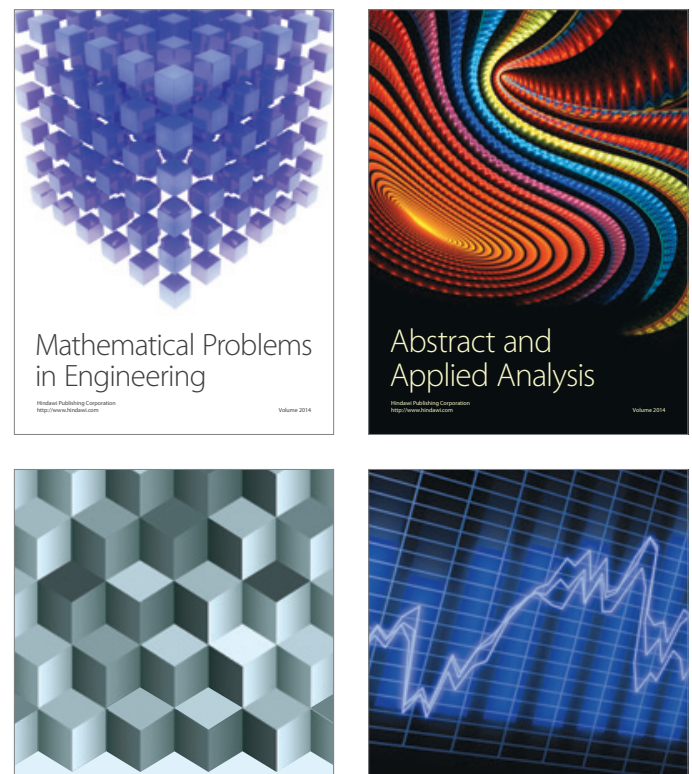

Journal of

Function Spaces

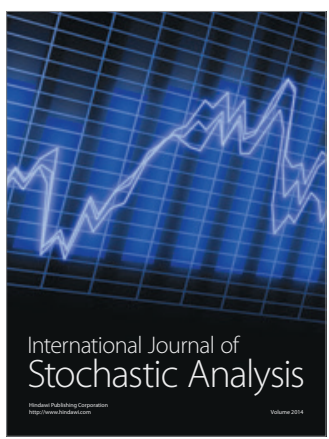

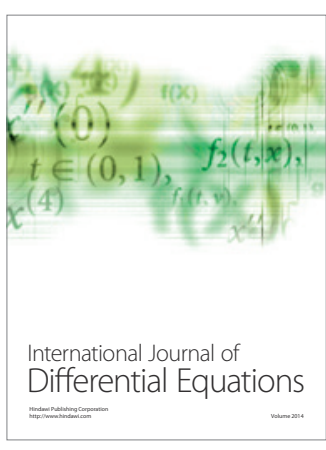
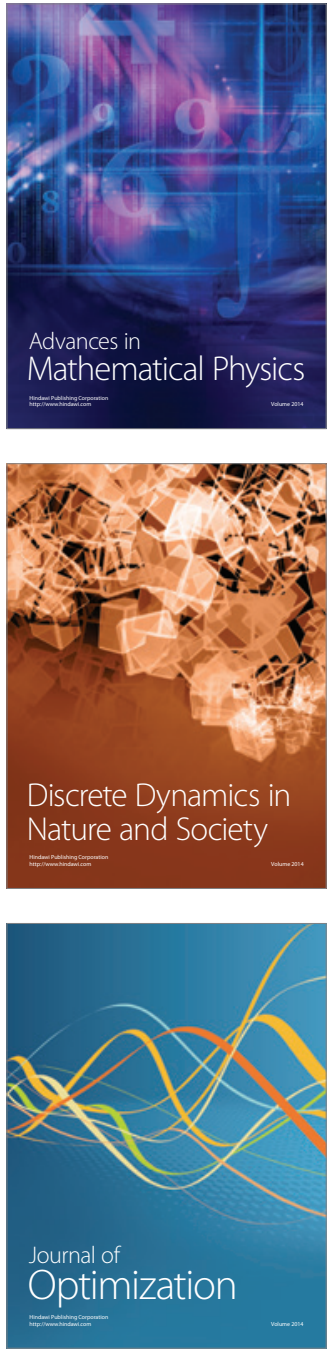